\title{
REVIEW
}

\section{Year in review 2009: Critical Care - nephrology}

\author{
Zaccaria Ricci*1 and Claudio Ronco ${ }^{2}$
}

\begin{abstract}
We summarize original research in the field of critical care nephrology accepted or published in 2009 in Critical Care or, when considered relevant or directly linked to this research, in other journals. Four main topics have been identified for a rapid overview: (a) post-surgical acute kidney injury (AKI); (b) timing of renal replacement therapy (RRT): different authors examined this critical issue of RRT in different settings (post-surgical patients, burned patients, and intensive care unit patients); (c) DoReMi (Dose Response Multicentre International) and other important surveys on dialysis dose and management; and (d) pediatric AKI and RRT: interest in this last topic is increasing, and studies on biomarkers, complications of pediatric dialysis, and application of RRT to extracorporeal membrane oxygenation are discussed.
\end{abstract}

\section{Post-surgical acute kidney injury}

Acute kidney injury (AKI) has been proven to increase patient mortality in all clinical settings: general out-ofhospital population, in-hospital admissions, adult and pediatric intensive care units (ICU), adult and pediatric cardiac surgery, and (last but not least) the relatively high portion formed by post-operative general surgery patients. In a study population of 1,166 patients without previous renal insufficiency, Abelha and colleagues [1] elegantly showed that $7.5 \%$ met AKI criteria. Interestingly, AKI was diagnosed when criteria of class I (or greater) of the Acute Kidney Injury Network (AKIN) classification were present. On multivariate analysis, American Society of Anesthesiologists (ASA) physical status, Revised Cardiac Risk Index (RCRI) score, high-risk surgery, and congestive heart failure were identified as the independent preoperative risk factors for AKI during the post-operative period. The RCRI score includes the following variables:

*Correspondence: z.ricci@libero.it

'Department of Pediatric Cardiosurgery, Bambino Gesù Hospital, Piazza S. Onofrio 400165 , Rome, Italy

Full list of author information is available at the end of the article high-risk surgery, ischemic heart disease, congestive heart failure, cerebrovascular disease, and insulinrequiring diabetes mellitus. According to these data, AKI patients were the most severely ill after ICU admission (higher Simplified Acute Physiology Score II and Acute Physiology and Chronic Health Evaluation II), had the longest ICU length of stay, and were independently at risk for hospital mortality. In our opinion, even if the accompanying editorial points out that one of the most important limitations of this report was the exclusion of patients with pre-operative renal dysfunction [2] (which has been identified as a major risk factor for perioperative AKI in most studies), patients with preoperative renal dysfunction are already those who receive the greater attention for prevention or treatment (or both) of further renal insult. So it must be remarked that an important message of this study is that post-operative AKI must be suspected in all patients with the clinical characteristics analyzed by Abelha and colleagues [1]. The next step will be to analyze such a cohort for the effect of intra-operative and post-operative therapeutic staregies on AKI risk: the prevention from use of nephrotoxins (nephrotoxic antibiotics, nonsteroidal antiinflammatory drugs, and some forms of hydroxyethyl starch), the effort to avoid extreme intra-operative hypotension or anemia, and finally the contribution of specifically targeted therapies (for example, bicarbonate infusion, N-acetilcysteine, fenoldopam, polymixin hemoperfusion, and prophylactic dialysis).

\section{Timing of renal replacement therapy}

The study by Abelha and colleagues [1] did not provide data on how many AKI patients underwent post-surgery renal replacement therapy (RRT). Shiao and colleagues [3] examined the impact of RRT timing in 98 patients affected by post-abdominal surgery AKI. The patients were divided according to RIFLE (Risk, Injury, Failure, Loss of function, End-stage kidney disease) classification into early dialysis (ED) (RIFLE-0 or Risk $=52 \%$ ) and late dialysis (LD) (RIFLE-Injury or Failure $=48 \%$ ). Fifty-seven patients (58.2\%) died during hospitalization; LD had a death hazard ratio (HR) of 1.846; other factors independently associated with risk of dying were old age (HR 2.090), cardiac failure (HR 4.620), and pre-RRT SOFA (Sequential Organ Failure Assessment) score (HR 1.152). 


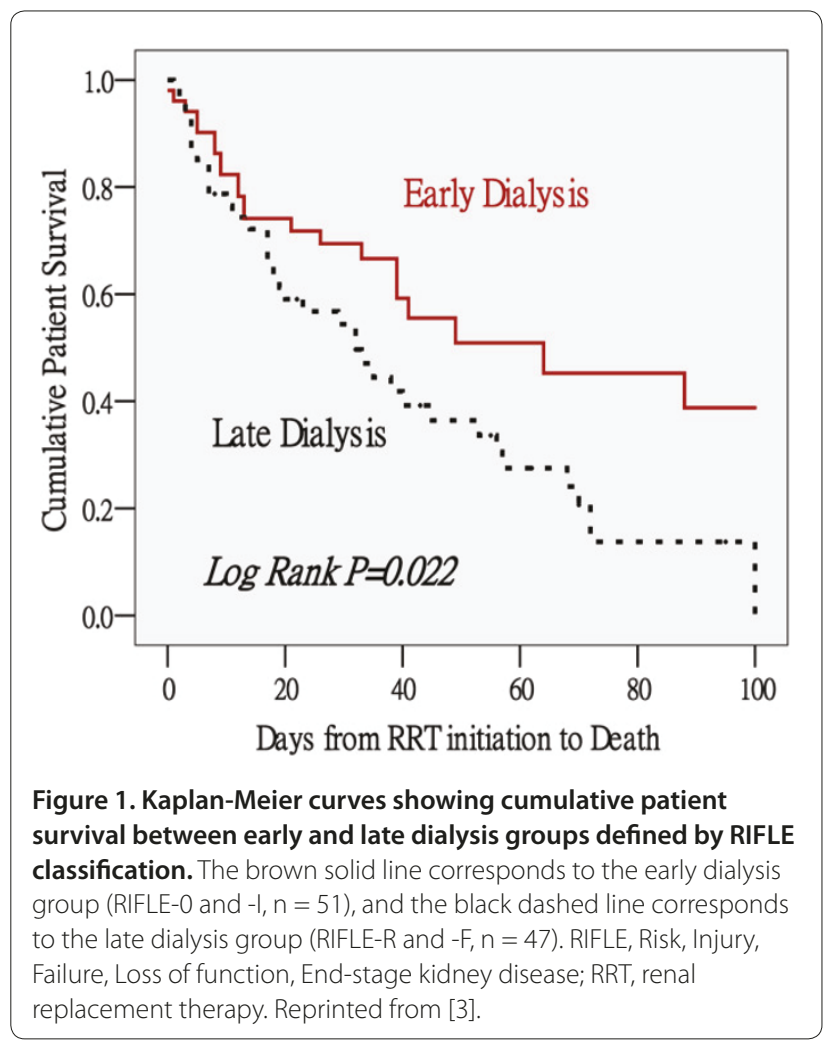

The findings of this study support earlier initiation of acute RRT (Figure 1). Interestingly, the authors used RIFLE classification as a prognostic tool in patients with post-major abdominal surgery AKI. However, defining ED and LD on the basis of RIFLE criteria may be only partially correct since AKI severity criteria do not necessarily indicate that the clinicians 'delayed' or 'anticipated' the dialytic therapy. (A RIFLE-F stage may occur and require RRT soon after ICU admission. Is this an LD?)

As a matter of fact, timing of RRT is crucial in AKI critically ill patients, and there is general agreement that a survival benefit is provided by early initiation of RRT. In clinical practice, however, to start early RRT remains quite a difficult choice. The differentiation between 'early' and 'late' RRT is based on arbitrary thresholds of traditional parameters such as serum urea, serum creatinine, urine output, time from ICU admission, or time from AKI diagnosis [4]. Furthermore, it may happen that RRT is indicated at an early ICU admission stage, whereas late initiation of renal support is prompted in an advanced phase of multiple organ dysfunction syndrome; the different clinical pictures of these two RRT prescriptions may not be classified simply as 'early' or 'late.' The detractors of a strategy of early initiation of RRT, finally, claim that patients who would recover renal function with conservative treatment alone may be subjected to unnecessary risks. Recently, in an interesting retrospective analysis of 1,847 critically ill patients with AKI requiring RRT, Ostermann and Chang [5] evaluated the relationship between biochemical, physiological, and comorbid factors at time of RRT and ICU mortality. Multivariate analysis showed that, at time of initiation of RRT, independent risk factors for ICU mortality were mechanical ventilation (odds ratio [OR] 6.03), neurological failure (OR 2.48), liver failure (OR 2.44), gastrointestinal failure (OR 2.04), pre-existing chronic illnesses (OR 1.74), hematological failure (OR 1.74), respiratory failure (OR 1.62), oligoanuria (OR 1.6), age (OR 1.03), serum urea (OR 1.004), and cardiovascular failure (OR 1.3). A higher $\mathrm{pH}$ at initiation of RRT was independently associated with a better outcome. Failure to correct acidosis and development of more organ failure within 48 hours after initiation of RRT were also associated with an increased risk of dying in the ICU. Even if these results come from a retrospective analysis and are, by definition, inconclusive, the message they carry seems to be that RRT should be commenced for AKI critically ill patients before organ failure and metabolic derangements have reached the slippery threshold of irreversibility. An interesting and controversial part of the paper concerns serum creatinine and urea concentrations on the day of RRT start. At RRT start, survivors tended to have lower urea and higher creatinine levels. This finding further suggests that the decision when to start RRT for AKI should be guided more by associated dysfunction of other organ systems, urine output, and serum $\mathrm{pH}$ than by absolute serum creatinine or urea levels (or both). Clearly, creatinine is not an ideal biomarker for decisions on RRT timing. Creatinine can result normal in the case of RRT for fluid overload (that decreased creatinine levels because of hemodilution) or 'extrarenal' RRT indications (a subgroup of patients with normal creatinine but still poor outcome). However, patients who received RRT before they met the creatinine criteria for AKIN stage III had a significantly lower ICU mortality than patients who were started on RRT on the day when they met the AKIN stage III criteria (49.8\% versus $64.6 \%$ ). The early start of RRT was recently supported by a retrospective cohort study that showed how initiating dialysis with a blood urea nitrogen of more than $100 \mathrm{mg} / \mathrm{dL}$ predicted death at 14 days (OR 3.6, 95\% confidence interval [CI] 1.7 to 7.6 ), 28 days (OR 2.6, 95\% CI 1.2 to 5.7 ), and 365 days (OR $3.5,95 \%$ CI 1.2 to 10 ) [6]. Though imperfect, biomarkers for RRT initiation are the simplest guide that clinicians commonly follow in clinical practice. In this light, the new biomarkers (see below) will hopefully improve the performance of creatinine and urea.

Last year, in the 'Year in review 2008: Critical Care-nephrology' [7], we commented on the work by Steinvall and colleagues [8], who analyzed AKI incidence in a 
cohort study of patients with burns to more than $20 \%$ of total body surface area (TBSA). Of these patients, $24 \%$ developed AKI and 3\% required dialysis. Interestingly, Steinvall and colleagues found that approximately one half of patients developed AKI during the first week and the other half developed AKI during the next week. Apparently, the authors' resuscitation protocol was successful in preventing AKI but only when renal injury occurred in the very early phase of ICU admission. In a 2009 study of a population of patients with TBSA burns of more than $40 \%$ and AKI, Chung and colleagues [9] aimed to determine the effect on mortality of early application of high-dose continuous venovenous hemofiltration $(\mathrm{CVVH})$ versus conservative management (fluid resuscitation, minimization of nephrotoxic agents, utilization of intermittent hemodialysis in case of refractory acidosis, electrolyte abnormalities, symptomatic fluid overload not responsive to conservative interventions, and intoxication with a dialyzable agent). The control group was formed by a historical cohort. AKI was diagnosed on the basis of AKIN criteria. The CVVH group was initiated on therapy $\left(\mathrm{T}_{0}\right)$ at a median of 9 days after admission, whereas the control group was diagnosed with AKI $\left(\mathrm{T}_{0}\right)$ at a median of 19 days after admission $(P=0.32)$. 'Early AKI', defined as the presence of AKI within 14 days from time of admission, occurred in $62 \%$ of patients in the CVVH group and $46 \%$ of patients in the control group $(P=0.24)$. Patients in the CVVH group were initially prescribed a mean hemofiltration dose of $57 \pm 19 \mathrm{~mL} / \mathrm{kg}$ per hour. The mean duration of treatment was $5.6 \pm 4.1$ days. The 28-day mortality was significantly lower in CVVH patients than in controls $(38 \%$ versus $71 \%, P=0.011)$ as was the inhospital mortality $(62 \%$ versus $86 \%, P=0.04)$. The authors also evaluated the effect of CVVH on multiple organ failure and showed that a significant decrease in vasopressor requirement and a significant increase in the ratio of partial pressure of arterial oxygen to fraction of inspired oxygen were seen in the CVVH group in comparison with controls. This study strongly encourages the use of early CVVH even in a peculiar setting such as that of burned patients. A randomized trial should now definitely confirm these results and overcome all of the limitations of matched controlled studies: as the authors acknowledge, the two populations had some small differences (in age, severity of disease, and time to AKI diagnosis) that might have favored the CVVH group. Furthermore, AKIN II patients were included in controls (whereas it looks like all AKI patients were treated by $\mathrm{CVVH}$ in the treatment group), and no information on how many controls were treated by intermittent hemodialysis is provided. It looks like the historic cohort was undertreated, and no conclusions on RRT modality and dose by this study can be drawn.

\section{DoReMi and the importance of surveying}

The Acute Dialysis Quality Initiative workgroup [10] recommended that researchers study technical aspects of RRT and worldwide utilization of different techniques in order to clarify which renal replacement technique or schedule (or combination of the two) might increase outcomes of critically ill patients. Hence, several surveys on management and practice of RRT have been conducted in recent years [11-15]. These studies depict 'real world' clinical practice patterns and their possible correlation with patient outcomes. A typical example of this kind of observational study is the Beginning and Ending Supportive Study (BEST). This is a multicenter, multinational, prospective, epidemiological study with the aim of elucidating different aspects of AKI worldwide. The study, conducted at 54 centers in 23 countries, lasted only one year and yielded information on about 1,700 AKI patients, of whom about 70\% required RRT. Several studies have been published after analysis of data provided by the survey, and six of them concerned technical aspects of RRT [16-21]. RRT results of the BEST study showed that continuous renal replacement therapy (CRRT) is often the preferred choice (80\%) over intermittent renal replacement therapy (IRRT) (20\%), probably because critically ill patients who receive CRRT are likely to be hypotensive and severely ill [16]. Nevertheless, it was shown among dialysis survivors that CRRT was an independent predictor of recovery from dialysis dependence at hospital discharge with respect to IRRT [17]. The median prescribed CRRT dose during the survey was $20 \mathrm{~mL} / \mathrm{kg}$ per hour. No technical CRRT feature (dose, modality, type of filter, or anticoagulation technique) seemed to correlate with mortality at multivariate logistic regression analysis [18]. Cost of RRT, according to BEST authors, is higher for continuous therapies with respect to intermittent dialysis. The cost difference is due primarily to the utilization of dialysis and replacement fluids: a dose prescription modification from 35 to $20-25 \mathrm{~mL} / \mathrm{kg}$ per hour and consequent decrease of fluid requirement might allow a significant saving of CRRT expense [19]. From the BEST database, it seemed that late RRT start, when considered as time from ICU admission, was associated with greater mortality [20]. Among CRRT patients, survival was around $50 \%$; $60 \%$ of survivors were successfully weaned from renal replacement (no RRT need for at least 7 days after dialysis interruption); when compared with the repeatRRT group' (those who failed weaning), these patients had lower mortality, lower creatinine concentration, and higher urine output at the time of CRRT discontinuation [21].

Another important observational study, the Dose Response Multicentre International (DoReMi) collaborative initiative, examined delivered RRT dose in patients 
enrolled at 30 ICUs of eight European countries [22]. Patients were treated with either CRRT or IRRT during their ICU stay. Data were entered by operators into electronic case forms on a web server. Dose was categorized as more intensive (CRRT at least $35 \mathrm{~mL} / \mathrm{kg}$ per hour, IRRT at least six sessions per week) or less intensive (CRRT less than $35 \mathrm{~mL} / \mathrm{kg}$ per hour, IRRT fewer than six sessions per week). The authors analyzed 553 AKI patients treated with RRT: 338 received CRRT only, 87 received IRRT only, and 128 received both forms of dialysis. Of note, only $22 \%$ of CRRT patients received a more intensive dose. As in the BEST study, no evidence emerged from the DoReMi study for a survival benefit afforded by higher-dose RRT: crude ICU mortality rates among intensive CRRT patients were $60.8 \%$ versus $52.5 \%$ in less intensive patients. In IRRT, this was $23.6 \%$ versus $19.4 \%$, respectively. On multivariable analysis, there was no significant association between RRT dose and ICU mortality. Among survivors, shorter ICU stay and duration of mechanical ventilation were observed in the more intensive RRT groups. Overall, the median prescribed CRRT dose was $34 \mathrm{~mL} / \mathrm{kg}$ per hour, and the median delivered dose was about $27 \mathrm{~mL} / \mathrm{kg}$ per hour. It might be that, in the clinical field, theoretic prescription schedules do not fit with practical problems encountered during continuous therapies; the most common causes for CRRT interruption were clotting of the circuit $(74 \%$ of episodes), vascular access problem (11\%), and clinical reasons (10\%). For IRRT, the median delivered dose was relatively high: seven sessions per week. In regard to the cost issue, it should now be evaluated whether the actual reduction of length of stay and reduced medical resources utilization (that is, mechanical ventilation), together with the possibility that CRRT improves renal recovery among AKI survivors, justify the utilization of such a relatively expensive therapy.

The results of the BEST and DoReMi studies do not seem to encourage or support the prescription and delivery of 'intense' RRT (that is, $35 \mathrm{~mL} / \mathrm{kg}$ per hour or more during continuous RRT) versus less intense RRT (that is, 20 to $25 \mathrm{~mL} / \mathrm{kg}$ per hour during continuous RRT). Two recent multicenter clinical trials - the randomized evaluation of normal versus augmented level (RENAL) replacement therapy study [23] and the Veterans Administration/National Institutes of Health (VA/NIH) Acute Renal Failure Trial Network (ATN) study [24] examined the impact of RRT dose on mortality in critically ill patients. Neither study showed a benefit in outcomes by increases in intensity of RRT dose. In the RENAL trial, when the post hoc analysis was focused on the subgroup of septic patients, there was a tendency to lower mortality with the higher intensity approach only (OR 0.84, 95\% CI 0.62 to 1.12). However, the definition of 'normal dose' should be re-evaluated and compared with standard clinical practice [25]. It must be considered that both trials were rigorous clinical trials and greatly minimized the discrepancy between prescribed and delivered doses. Hence, in clinical practice, when $20 \mathrm{~mL} / \mathrm{kg}$ per hour is prescribed during continuous RRT (consistently with those in the RENAL and ATN studies), the possibility of a significant reduction in dialysis dose delivery should be considered. As clearly shown by DoReMi, when clinicians prescribe RRT, they must consider a $25 \%$ safety margin, targeting 25 to $30 \mathrm{~mL} / \mathrm{kg}$ per hour in order to meet the actual delivered dose of 19 to $22 \mathrm{~mL} / \mathrm{kg}$ per hour [25].

\section{Pediatric acute kidney injury and renal replacement therapy}

In recent years, application of AKI knowledge from the adult critically ill patients to the pediatric setting has revealed a new and interesting field of research. In particular, cardiac surgery-associated AKI is a convenient clinical setting for the study of early AKI biomarkers since there is a temporally predictable insult to the kidneys and since it is possible to measure urine and blood levels of these biomarkers before the actual injury and compare them with levels at pre-specified time points afterwards. The NGAL (Neutrophil GelatinaseAssociated Lipocaline) Meta-analysis Investigator Group recently published the results of the analysis of data from 19 studies and 8 countries; the data involved 2,538 patients, of whom 487 (19.2\%) developed AKI [26]. The authors found that NGAL levels clearly appeared to be of diagnostic and prognostic value for AKI, RRT, and mortality, especially in cardiac surgery patients and in children. Levels of serum interleukin (IL)-1-beta, IL-5, IL-6, IL-8, IL-10, IL-17, interferon-gamma, tumor necrosis factor-alpha, granulocyte colony-stimulating factor (G-CSF), and granulocyte-macrophage colonystimulating factor (GM-CSF) as early biomarkers of AKI were also measured in a case control study of children undergoing cardiac surgery (18 cases and 21 controls) [27]. AKI was defined as a $50 \%$ increase in serum creatinine from baseline within 3 days. IL-6 levels at 2 and 12 hours after cardiopulmonary bypass and IL-8 levels at 2, 12, and 24 hours were associated with the development of AKI. In patients with AKI, IL-6 levels at 2 hours had excellent predictive value for prolonged mechanical ventilation (defined as mechanical ventilation for more than 24 hours post-operatively) by receiver operator curve (ROC) analysis, with an area under the ROC of 0.95. IL-8 levels at 2 hours had excellent predictive value for prolonged mechanical ventilation in all patients. Serum IL-18 levels between subjects with AKI and those without AKI were not different. A panel of several AKI biomarkers, similar to those in ischemic heart disease diagnosis, is expected in the future in order 
to diagnose, prevent, and possibly treat AKI and its complications.

Possibly owing to the lack of specifically designed devices, pediatric AKI requiring RRT is currently managed with a high occurrence of side effects in many centers. Santiago and colleagues [28] prospectively analyzed complications during CRRT in 174 critically ill children over a 13-year period. Of the studied patients, a relatively low percentage $(7.4 \%)$ presented problems of venous catheterization (hematoma at the puncture site, hemorrhage, altered venous drainage of the lower limbs, and incorrect position of the jugular venous catheter requiring change). Hypotension at CRRT start was detected in one third of patients. Clinically significant hemorrhage occurred in $10 \%$ of patients. In the first 72 hours of CRRT, the levels of sodium, chloride, and phosphate fell significantly; total calcium increased significantly; and the levels of potassium and magnesium remained unaltered; the changes in electrolyte levels during the course of treatment were not associated with mortality. This study, the first large analysis of the complication of pediatric CRRT, finds that complications in this cohort of patients are still high and may be greater than in adults. The historical observational nature of the study design does not allow any definitive conclusion to be drawn and some questions are left unanswered. For example, are adult RRT materials safe and effective when adapted to children and newborn patients?

However, experience with pediatric CRRT is increasing and improved technical features of 'pediatric-adapted' dialysis machines warrant safer treatments. In particular, a peculiar and complex category of pediatric patient is the infant with multiple organ dysfunction, requiring both RRT and extracorporeal membrane oxygenation (ECMO). AKI occurs to the vast majority of ECMO children, who suffer from severe cardiac dysfunction (cardio-renal syndrome) or required aggressive mechanical ventilation (lung-renal syndrome). The CRRT circuit is placed in parallel (blood flows in the same direction as the ECMO circuit) or in series (countercurrent to the ECMO circuit). Santiago and colleagues [29] described how to connect the CRRT device to the ECMO circuit: the inlet (arterial) line of the CRRT circuit was connected after the ECMO blood pump by a threeway tap that was also used for the infusion of heparin, and the outlet (venous) line was connected to the circuit at another tap before the oxygenator. In contrast to what was suggested by the authors, the inlet of the CRRT machine may be connected after the ECMO pump and the filter outlet then returned to the ECMO circuit before the pump (into the reservoir, if present); the CRRT circuit, running countercurrent to extracorporeal assistance, allows the blood to be infused into the venous ECMO section (where the patient is drained) and then to be aspired from the arterial ECMO section (where blood returns to the patient) [30]. This second set-up might reduce blood flow resistance and turbulence after the centrifugal pump and improve reservoir drainage when a roller pump is present. The blood recirculation induced by these circuit set-ups is negligible, considering that the ratio of CRRT to ECMO blood flow is never greater than 0.1 . Shaheen and colleagues [31] recently reported their experience with two different subgroups of children: one that required hemofiltration alone and one that required hemofiltration and ECMO. Not surprisingly, the authors identified a higher mortality rate in those patients requiring $\mathrm{CVVH}$ and $\mathrm{ECMO}$ compared with those patients requiring hemofiltration alone. The authors promoted the concept that certain therapies should be reserved for experienced teams. Performing CVVH in a heterogeneous population with large ranges of age and weight poses significant clinical and technical challenges. The low frequency of CVVH use, as well as the use of other extracorporeal therapies, also raises problems with maintaining nursing skills. Objective clinical and biochemical markers for commencing CVVH alone or in combination with ECMO remain to be defined. Several studies, however, already showed safety and feasibility of this connection in the pediatric setting [32], and even if concerns about such difficult interaction have been raised (that is, fluid balance accuracy [33]), the application of CRRT to all ECMO patients is claimed by some authors [34]. In 15 patients matched with 46 historical controls, it has been shown that adding continuous hemofiltration to the ECMO circuit in newborns improves outcome by significantly reducing time on extracorporeal assistance and on mechanical ventilation. Such a strategy might improve fluid balance management and capillary leak syndrome. Furthermore, according to these authors, fewer blood transfusions are needed and overall costs per ECMO run are lower.

\section{Abbreviations \\ AKI, acute kidney injury; AKIN, Acute Kidney Injury Network; ATN, Acute Renal Failure Trial Network; BEST, Beginning and Ending Supportive Study; $\mathrm{Cl}$, confidence interval; CRRT, continuous renal replacement therapy; $\mathrm{CVVH}$, continuous venovenous hemofiltration; DoReMi, Dose Response Multicentre International; ECMO, extracorporeal membrane oxygenation; ED, early dialysis; $H R$, hazard ratio; ICU, intensive care unit; IL, interleukin; IRRT, intermittent renal replacement therapy; LD, late dialysis; NGAL, Neutrophil Gelatinase- Associated Lipocaline; OR, odds ratio; RCRI, Revised Cardiac Risk Index; RENAL, randomized evaluation of normal versus augmented level; RIFLE, Risk, Injury, Failure, Loss of function, End-stage kidney disease; ROC, receiver operator curve; RRT, renal replacement therapy; TBSA, total body surface area.}

Competing interests

The authors declare that they have no competing interests.

\section{Author details}

'Department of Pediatric Cardiosurgery, Bambino Gesù Hospital, Piazza S. Onofrio 4 00165, Rome, Italy. ${ }^{2}$ Department of Nephrology, Dialysis and Transplantation, S. Bortolo Hospital, Viale Rodolfi 36100, Vicenza, Italy. 
Published: 5 November 2010

\section{References}

1. Abelha FJ, Botelho M, Fernandes V, Barros H: Determinants of postoperative acute kidney injury. Crit Care 2009, 13:R79.

2. Murray $P$ : Who is at increased risk for acute kidney injury following noncardiac surgery? Crit Care 2009, 13:171.

3. Shiao CC, Wu VC, Li WY, Lin YF, Hu FC, Young GH, Kuo CC, Kao TW, Huang DM, Chen YM, Tsai PR, Lin SL, Chou NK, Lin TH, Yeh YC, Wang CH, Chou A, Ko WJ, Wu KD; National Taiwan University Surgical Intensive Care Unit-Associated Renal Failure Study Group: Late initiation of renal replacement therapy is associated with worse outcomes in acute kidney injury after major abdominal surgery. Crit Care 2009, 13:R171.

4. Uchino S: What is 'BEST'RRT practice? Contrib Nephrol 2010, 165:244-250.

5. Ostermann M, Chang RW: Correlation between parameters at initiation of renal replacement therapy and outcome in patients with acute kidney injury. Crit Care 2009, 13:R175.

6. Carl DE, Grossman C, Behnke M, Sessler CN, Gehr TW: Effect of timing of dialysis on mortality in critically ill, septic patients with acute renal failure. Hemodial Int 2010, 14:11-17.

7. Ricci Z, Ronco C: Year in review 2008: Critical Care-nephrology. Crit Care 2009, 13:22

8. Steinvall I, Bak Z, Sjoberg F: Acute kidney injury is common, parallels organ dysfunction or failure, and carries appreciable mortality in patients with major burns: a prospective exploratory cohort study. Crit Care 2008, 12:R124.

9. Chung KK, Lundy JB, Matson JR, Renz EM, White CE, King BT, Barillo DJ, Jones JA, Cancio LC, Blackbourne LH, Wolf SE: Continuous venovenous hemofiltration in severely burned patients with acute kidney injury: a cohort study. Crit Care 2009, 13:R62

10. Acute Dialysis Quality Initiative homepage [www.adqi.net]

11. Ricci Z, Ronco C, D'Amico G, De Felice R, Rossi S, Bolgan I, Bonello M, Zamperetti N, Petras D, Salvatori G, Dan M, Piccinni P: Practice patterns in the management of acute renal failure in the critically ill patient: an international survey. Nephrol Dial Transplant 2006, 21:690-696.

12. Mariano F, Pozzato M, Canepari G, Vitale C, Bermond F, Sacco C, Amore A Manes M, Navino C; Piedmont and aosta Valley Section of Italian Society of Nephrology: Renal replacement therapy in intensive care units: a survey of nephrological practice in northwest Italy. J Nephrol 2010 Jun 14 [Epub ahead of print].

13. Payen D, de Pont AC, Sakr Y, Spies C, Reinhart K, Vincent JL; Sepsis Occurrence in Acutely III Patients (SOAP) Investigators: A positive fluid balance is associated with a worse outcome in patients with acute renal failure. Crit Care 2008, 12:R74.

14. Overberger P, Pesacreta M, Palevsky PM; VA/NIH Acute Renal Failure Trial Network: Management of renal replacement therapy in acute kidney injury: a survey of practitioner prescribing practices. Clin J Am Soc Nephro 2007, 2:623-630

15. Symons JM, Chua AN, Somers MJ, Baum MA, Bunchman TE, Benfield MR Brophy PD, Blowey D, Fortenberry JD, Chand D, Flores FX, Hackbarth R, Alexander SR, Mahan J, McBryde KD, Goldstein SL: Demographic characteristics of pediatric continuous renal replacement therapy: a report of the prospective pediatric continuous renal replacement therapy registry. Clin J Am Soc Nephrol 2007, 2:732-738.

16. Uchino S, Kellum JA, Bellomo R, Doig GS, Morimatsu H, Morgera S, Schetz M, Tan I, Bouman C, Macedo E, Gibney N, Tolwani A, Ronco C; Beginning and Ending Supportive Therapy for the Kidney (BEST Kidney) Investigators: Acute renal failure in critically ill patients: a multinational, multicenter study. JAMA 2005, 294:813-818.

17. Uchino S, Bellomo R, Kellum JA, Morimatsu H, Morgera S, Schetz MR, Tan I, Bouman C, Macedo E, Gibney N, Tolwani A, Oudemans-Van Straaten HM, Ronco C; Beginning and Ending Supportive Therapy for the Kidney (B.E.S.T. Kidney) Investigators Writing Committee: Patient and kidney survival by dialysis modality in critically ill patients with acute kidney injury. Int J Artif Organs 2007, 30:281-292

18. Uchino S, Bellomo R, Morimatsu H, Morgera S, Schetz M, Tan I, Bouman C, Macedo E, Gibney N, Tolwani A, Oudemans-van Straaten H, Ronco C, Kellum
JA: Continuous renal replacement therapy: a worldwide practice survey The beginning and ending supportive therapy for the kidney (B.E.S.T. kidney) investigators. Intensive Care Med 2007, 33:1563-1570.

19. Srisawat N, Lawsin L, Uchino S, Bellomo R, Kellum JA; BEST Kidney Investigators: Cost of acute renal replacement therapy in the intensive care unit: results from The Beginning and Ending Supportive Therapy for the Kidney (BEST Kidney) study. Crit Care 2010, 14:R46.

20. Bagshaw SM, Uchino S, Bellomo R, Morimatsu H, Morgera S, Schetz M, Tan I, Bouman C, Macedo E, Gibney N, Tolwani A, Oudemans-van Straaten HM, Ronco C, Kellum JA; Beginning and Ending Supportive Therapy for the Kidney (BEST Kidney) Investigators: Timing of renal replacement therapy and clinical outcomes in critically ill patients with severe acute kidney injury. J Crit Care 2009, 24:129-140.

21. Uchino S, Bellomo R, Morimatsu H, Morgera S, Schetz M, Tan I, Bouman C, Macedo E, Gibney N, Tolwani A, Straaten HO, Ronco C, Kellum JA: Discontinuation of continuous renal replacement therapy: a post hoc analysis of a prospective multicenter observational study. Crit Care Med 2009, 37:2576-2582.

22. Vesconi S, Cruz DN, Fumagalli R, Kindgen-Milles D, Monti G, Marinho A, Mariano F, Formica M, Marchesi M, René R, Livigni S, Ronco C; DOse REsponse Multicentre International collaborative Initiative (DO-RE-MI Study Group): Delivered dose of renal replacement therapy and mortality in critically ill patients with acute kidney injury. Crit Care 2009, 13:R57.

23. RENAL Replacement Therapy Study Investigators: Intensity of continuous renal-replacement therapy in critically ill patients. N Engl J Med 2009, 361:1627-1638.

24. VA/NIH Acute Renal Failure Trial Network: Intensity of renal support in critically ill patients with acute kidney injury. N Engl J Med 2008, 359:7-20

25. Kellum JA, Ronco C: Dialysis: Results of RENAL--what is the optimal CRRT target dose? Nat Rev Nephro/ 2010, 6:191-192

26. Haase M, Bellomo R, Devarajan P, Schlattmann P, Haase-Fielitz A; NGAL Metaanalysis Investigator Group: Accuracy of neutrophil gelatinase-associated lipocalin (NGAL) in diagnosis and prognosis in acute kidney injury: a systematic review and meta-analysis. Am J Kidney Dis 2009, 54:1012-1024.

27. Liu KD, Altmann C, Smits G, Krawczeski CD, Edelstein CL, Devarajan P, Faubel S: Serum interleukin- 6 and interleukin- 8 are early biomarkers of acute kidney injury and predict prolonged mechanical ventilation in children undergoing cardiac surgery: a case-control study. Crit Care 2009, 13:R104.

28. Santiago MJ, López-Herce J, Urbano J, Solana MJ, del Castillo J, Ballestero Y, Botrán M, Bellón JM: Complications of continuous renal replacement therapy in critically ill children: a prospective observational evaluation study. Crit Care 2009, 13:R184.

29. Santiago MJ, Sánchez A, López-Herce J, Pérez R, del Castillo J, Urbano J, Carrillo A: The use of continuous renal replacement therapy in series with extracorporeal membrane oxygenation. Kidney Int 2009, 76:1289-1292.

30. Ricci Z, Ronco C, Picardo S: CRRT in series with extracorporeal membrane oxygenation in pediatric patients. Kidney Int 2010, 77:469-470.

31. Shaheen IS, Harvey B, Watson AR, Pandya HC, Mayer A, Thomas D: Continuous venovenous hemofiltration with or without extracorporeal membrane oxygenation in children. Pediatr Crit Care Med 2007, 8:362-365

32. Hoover NG, Heard M, Reid C, Wagoner S, Rogers K, Foland J, Paden ML, Fortenberry JD: Enhanced fluid management with continuous venovenous hemofiltration in pediatric respiratory failure patients receiving extracorporeal membrane oxygenation support. Intensive Care Med 2008, 34:2241-2247.

33. Ricci Z, Morelli S, Vitale V, Di Chiara L, Cruz D, Picardo S: Management of fluid balance in continuous renal replacement therapy: technical evaluation in the pediatric setting. Int J Artif Organs 2007, 30:896-901.

34. Blijdorp K, Cransberg K, Wildschut ED, Gischler SJ, Jan Houmes R, Wolff ED Tibboel D: Haemofiltration in newborns treated with extracorporeal membrane oxygenation: a case-comparison study. Crit Care 2009, 13:R48.

doi:10.1186/cc9277

Cite this article as: Ricci Z, Ronco C : Year in review in Critical Care, 2009:

Nephrology. Critical Care 2010, 14:241. 